\title{
Cirugía percutánea del pie: ¿hacia dónde debemos ir?
}

La cirugía percutánea del pie, también conocida como cirugía MIS (del inglés minimal incision surgery), es un método quirúrgico que permite realizar intervenciones a través de incisiones mínimas, sin exposición directa de los planos quirúrgicos. Esta técnica ocasiona un trauma mínimo de los tejidos próximos y precisa un control radiológico durante la intervención, para orientarse en los gestos quirúrgicos que se deben realizar. Sin bien esta es la definición "de libro", debemos considerar algunos puntos adicionales para describirla.

Este procedimiento se encuentra, en la actualidad, en fase de estabilización-evolución, acompañando lenta y progresivamente a la cirugía convencional [abierta] y reduciendo la morbilidad, dando como resultado un porcentaje creciente de éxitos quirúrgicos.

De acuerdo con los estudios publicados, se pueden diferenciar tres grupos de procedimientos: en primer lugar, se encuentran los "procedimientos aceptados" y considerados como una opción razonable; en segundo lugar, se encuentran aquellos procedimientos que han demostrado ser técnicamente factibles de realizar, pero sin aceptación amplia por la comunidad quirúrgica; y en tercer lugar, se incluyen aquellas operaciones "no aceptadas actualmente" (Tabla).

Antes de responder la pregunta que da lugar a esta reflexión, es necesario detenerse sobre algunas cuestiones que requieren mayor atención y que son dignas de continuar creciendo:

- Deficiencias en la formación académica: si bien el Grecmip (Groupe de Recherche et d'Etude en Chirurgie Mini-Invasive $d u$ Pied) y otras sociedades científicas han impulsado, en la última década, congresos y cursos de formación cadavéricos, aún sigue siendo insuficiente la instalación y divulgación de la técnica en actividades científicas nacionales o continentales. Esto conlleva a enlentecer la formación de los colegas que así lo deseen.

- Falta de profundidad al medir resultados: aunque existen estudios sobre resultados clínicos buenos, muy buenos o excelentes, todavía no se han tratado con mayor detenimiento casos de complicaciones, infecciones profundas y superficiales o trabajos comparativos de resultados a largo plazo.

- Diseño de trabajos científicos: se conoce la complejidad que conlleva la redacción de trabajos de investigación, a la cual se le suma un predador sistema de publicación formado por revistas ávidas de contenido que luego no tienen repercusión. Sin embargo, algunas revistas científicas ya han incorporado análisis microeconómicos (principalmente costo-efectividad y costoutilidad) con sus respectivos niveles de evidencia. Los análisis de costo-utilidad se construyen con información sustraída de cuestionarios de calidad de vida (SF-36 y EQ-5L-5D) y, como resultado final, muestran indicadores como los QALY (QualityAdjusted Life-Years) o años de vida ajustados por calidad. Es importante tener en cuenta esta variante de trabajos en la actualidad, con el propósito de enriquecer la discusión.

Sería imprudente aseverar que la cirugía percutánea del pie va a reemplazar definitivamente al procedimiento tradicional. Si bien esta técnica ha reducido considerablemente el tiempo quirúrgico y la exposición de partes blandas, en un marco aún parcialmente teórico, es posible sugerir que podría traer menores complicaciones y perpetuar los resultados satisfactorios a través del tiempo. Sería recomendable que, con estirpe segura y firme, siga los pasos de la artroscopia en la cirugía de rodilla y posteriormente en las de tobillo y cadera: primero sólo lo realizaban unos pocos que eran considerados aventureros, pasando por una mirada esperanzada hasta llegar a la aceptación completa y definitiva de la población de cirujanos y sociedades científicas.

Por último, cabe destacar que nuestro deber como médicos es intentar "empujar" la medicina hacia adelante, ya que debemos recordar que el paciente es el bien último y necesita de las mejores herramientas.

"La madre del conocimiento es la ciencia; la opinión genera ignorancia."

Hipócrates [460-370 a.C.] 


\section{Bibliografía consultada y recomendada}

1. Bauer T, de Lavigne C, Biau D, De Prado M, Isham S, Laffenétre O. Percutaneous hallux valgus surgery: a prospective multicenter study of 189 cases. Orthop Clin North Am 2009;40(4):505-14.

2. Bersusky E. Las editoriales predadoras. Rev Asoc Argent Ortop Traumatol 2017;82(2):89-90.

3. Bosch P, Wanke S, Legenstein R. Hallux valgus correction by the method of Bosch: a new technique with a seven-to-ten-year follow-up. Foot Ankle Clin 2000;5:485-98, v-vi.

4. Dohmen L. ¿Por qué nos cuesta escribir artículos científicos? Rev Asoc Argent Ortop Traumatol 2017;82(Supl. 1):1.

5. De Prado M, Ripoll PL, Golano P. Cirugía percutánea del pie: técnicas quirúrgicas, indicaciones, bases anatómicas. Barcelona: Masson; 2003.

6. del Vecchio JJ, Ghioldi ME, Raimondi N. Osteotomía en tejadillo (Chevron) con técnica mínimamente invasiva en la región distal del primer metatarsiano. Evaluación radiológica. Rev Asoc Argent Ortop Traumatol 2017;82(1):19-27.

7. Edlin R, McCabe C, Hulme C, Hall P, Wright J. Cost effectiveness modeling for health technology assessment. A practical course. Switzerland: Springer International Publishing; 2015.

8. Elmallah RK, Chughtai M, Adib F, Bozic KJ, Kurtz SM, Mont MA. Determining health-related quality-of-life outcomes using the SF-6D following total hip arthroplasty. J Bone Joint Surg Am 2017;15;99(6):494-8.

9. Haque S, Kakwani R, Chadwick C, Davies MB, Blundell CM. Outcome of minimally invasive distal metatarsal metaphyseal osteotomy (DMMO) for lesser toe metatarsalgia. Foot Ankle Int 2016;37(1):58-63.

10. http://panelfenin.es/uploads/fenin/documento_estudios/pdf_documento_18.pdf.

11. Lucas y Hernandez J, Golanó P, Roshan-Zamir S, Darcel V, Chauveaux D, Laffenêtre O. Treatment of moderate hallux valgus by percutaneous, extra-articular reverse-L Chevron (PERC) osteotomy. Bone Joint J Br 2016;98(3):365-73.

12. Lee M, Walsh J, Smith MM, Ling J, Wines A, Lam P. Hallux valgus correction comparing percutaneous Chevron/Akin (PECA) and open scarf/Akin osteotomies. Foot Ankle Int 2017;38(8):838-46.

13. Osterhoff G, O'Hara NN, D’Cruz J, Sprague SA, Bansback N, Evaniew N, et al. A cost-effectiveness analysis of reverse total shoulder arthroplasty versus hemiarthroplasty for the management of complex proximal humeral fractures in the elderly. Value Health 2017;20(3):404-11.

14. http://www.grecmip.org/.

15. Yañez Arauz JM, Del Vecchio JJ, Raimondi N, Codesido MA. Riesgo de lesiones quirúrgicas en la fasciotomía plantar percutánea. Estudio anatómico en cadáveres frescos. Rev Asoc Argent Ortop Traumatol 2011;76(2):141-5.

16. Yañez Arauz JM, Del Vecchio JJ, Codesido M, Raimondi N. Minimally invasive Akin osteotomy and lateral release: anatomical structures at risk-A cadaveric study. Foot (Edinb) 2016;27:32-5.

Tabla. Grupos de procedimientos según grados de aceptación

\begin{tabular}{|c|c|c|}
\hline $\begin{array}{l}\text { Procedimientos } \\
\text { aceptados }\end{array}$ & $\begin{array}{l}\text { Procedimientos factibles } \\
\text { de posible aceptación }\end{array}$ & $\begin{array}{l}\text { Procedimientos no aceptados } \\
\text { actualmente }\end{array}$ \\
\hline Osteotomía de Reverdin-Isham & MICA (Minimally invasive Chevron-Akin) & Artrodesis de tobillo, retro y mediopié \\
\hline Osteotomía de Bösch & $\begin{array}{l}\text { PERC (Percutaneous, extra-articular } \\
\text { reverse-L Chevron) }\end{array}$ & Artrodesis de hallux \\
\hline $\begin{array}{l}\text { DMMO (Distal metatarsal minimally } \\
\text { invasive osteotomy) }\end{array}$ & PECA (Percutaneous Chevron/Akin) & Revisiones de cirugía abierta de antepié \\
\hline $\begin{array}{l}\text { Osteotomía de Akin aislada o en } \\
\text { combinación con cirugía abierta ("híbrida") }\end{array}$ & $\begin{array}{l}\text { Osteotomía de base de primer } \\
\text { metatarsiano }\end{array}$ & Osteotomía de retro y mediopié \\
\hline Liberación lateral por vía lateral & $\begin{array}{l}\text { PICO (Percutaneous, intra-articular, } \\
\text { Chevron osteotomy) }\end{array}$ & Sesamoidectomías totales o parciales \\
\hline Tenotomías flexo-extensoras & Osteotomía DPR (De Prado) & Resección de exostosis de Haglund \\
\hline Exostectomías & S-DMMO (Sliding DMMO) & Minimally invasive scarf \\
\hline Capsulotomía & $\begin{array}{l}\text { Osteotomía de primera falange } \\
\text { de dedos menores }\end{array}$ & \\
\hline Fasciotomía plantar total/subtotal & & \\
\hline
\end{tabular}

